\title{
Erythropoietin Protects Dopaminergic Neurons and Improves Neurobehavioral Outcomes in Juvenile Rats after Neonatal Hypoxia-Ischemia
}

\author{
ERIC J. DEMERS, RONALD J. MCPHERSON, AND SANDRA E. JUUL \\ Department of Pediatrics, University of Washington, Seattle, Washington 98195
}

\begin{abstract}
Brain injury as a result of hypoxia-ischemia remains a common cause of morbidity and mortality in neonates. No effective therapy is currently available. The hematopoietic cytokine erythropoietin (Epo) provides neuroprotection in many adult models of brain injury and is currently being investigated as a therapeutic agent for human stroke and spinal cord injury. We tested the hypothesis that recombinant Epo (rEpo) would improve neurobehavioral outcomes after neonatal hypoxic-ischemic brain injury. Postnatal day 7 rats underwent right common carotid artery occlusion followed by a $90-\mathrm{min}$ exposure to $8 \%$ oxygen. Rats were subsequently treated with rEpo or placebo. Sensory neglect and apomorphine-induced rotation were measured at P27 and P28. Rats were killed at P30, blood was drawn, and the brains were perfusion-fixed for histology and immunohistochemistry. No differences in gross brain injury between rEpo and placebotreated rats were found. Neonatal rEpo treatment protected dopamine neurons as indicated by the preservation of tyrosine hydroxylase-positive cells in the substantia nigra pars compacta and ventral tegmental area. rEpo treatment also improved functional outcomes by reducing sensory neglect and preventing the rotational asymmetry seen in control animals. No differences in
\end{abstract}

\section{ABSTRACT}

hematocrit, white blood cell counts, neutrophil counts, or platelet counts were measured. We observed that rEpo treatment protected mesencephalic dopamine neurons and reduced the degree of behavioral asymmetries at 4 wk of life. On the basis of these findings, we conclude that further studies investigating the safety and efficacy of high-dose rEpo as a neuroprotective strategy are indicated in neonatal models of hypoxic-ischemic brain injury.

(Pediatr Res 58: 297-301, 2005)

\section{Abbreviations}

ADHD, attention-deficit/hyperactivity disorder

DA, dopamine

Epo, erythropoietin

EpoR, erythropoietin receptor

HI, hypoxia-ischemia

$\mathbf{P}$, postnatal day

rEpo, recombinant Epo

SNpc, substantia nigra pars compacta

TH, tyrosine hydroxylase

VTA, ventral tegmental area
Perinatal exposure to hypoxia-ischemia $(\mathrm{HI})$ produces brain injury that is a significant source of morbidity and mortality for preterm and term neonates. Neonatal HI exposure initiates a multifactorial cascade that can persist for days, producing injury to multiple brain regions with corresponding motor, behavioral, and cognitive impairments. Although there are currently no effective therapies to ameliorate hypoxic-ischemic brain injury, several useful rodent models of neonatal brain injury are available. Using 7-d-old (P7) rat pups, which are comparable to near-term humans in brain maturity (1), the technique entails unilateral ligation of the common carotid

Received July 26, 2004; accepted December 8, 2004.

Correspondence: Sandra Juul, M.D., Ph.D., Department of Pediatrics, Division of Neonatology, University of Washington, Box 356320, Seattle, WA 98195; e-mail: sjuul@u.washington.edu.

Supported by National Institutes of Health (National Institute of Child Health and Human Development) Grant HD042213-01.

DOI: 10.1203/01.PDR.0000169971.64558.5A artery and subsequent exposure of the animals to hypoxia (Rice-Vannucci model) (2). This procedure produces permanent unilateral brain injury in multiple regions, with specific impact on the basal ganglia including decreases in striatal dopamine (DA) receptors and increases in striatal DA transporters $(3,4)$. Disruption of dopaminergic neurotransmission is suspect for several developmental disorders. Moreover, candidate neuroprotective treatments may be tested by examining specific markers of DA and dopaminergic function.

Erythropoietin (Epo) is a hematopoietic cytokine that has been shown to have beneficial effects in the central and peripheral nervous system (5-7). Epo receptors (EpoR) are present on many cell types in brain, and their activation by Epo results in cell-specific effects (8). Stimulation of neurons with recombinant Epo (rEpo) decreases glutamate toxicity (both $\mathrm{N}$-methyl-D-aspartate and $\alpha$-amino-3-hydroxy-5-methyl-4isoxazolepropionic acid mediated) $(9,10)$, up-regulates antiapoptotic factors Bcl- $\mathrm{x}_{\mathrm{L}}$ and Bcl-2 (4,11), and decreases nitric 
oxide-mediated injury (12-14). In addition, rEpo preserves blood-brain barrier integrity (15), enhances proliferation/ differentiation of oligodendrocyte precursors (16), and promotes neurogenesis from neural stem cells (17). rEpo has also been shown to promote the differentiation of DA neurons and stimulate DA release from the striatum $(18-20)$. The combination of these factors leads to decreased apoptosis and neurologic injury in various animal models, including HI-induced brain injury, kainate-induced seizures, experimental autoimmune encephalomyelitis, and concussive brain and spinal cord injury (21).

Here, we report results of an experiment that was designed to test for improved neurobehavioral outcomes tested $3 \mathrm{wk}$ after Epo treatment and neonatal HI exposure. Because the brain injury produced using this HI model affects multiple tissues, including (but not limited to) DA-containing pathways, we sought to identify possible behavioral asymmetries by borrowing indices of unilateral DA depletion used in the rodent model of Parkinson's disease. We hypothesized that unilateral $\mathrm{HI}$-induced brain injury might produce contralateral sensory neglect and DA agonist-stimulated rotation and that Epo treatment would reduce the degree of asymmetry in these indices. Recognizing that mesencephalic dopaminergic neurons of the substantia nigra pars compacta ( $\mathrm{SNpc}$ ) and ventral tegmental area (VTA) have a relatively high rate of apoptosis during development, are influenced by striatal injury, and can be protected by interventions that up-regulate Bcl-2 (22), we further hypothesized that rEpo treatment after neonatal HI exposure would protect DA neurons in both the SNpc and the VTA.

\section{METHODS}

Animals, injury, and treatment. This study was performed in accordance with the guidelines established by the Animal Care Committee at the University of Washington. The right common carotid artery of P7 Sprague-Dawley rat pups was isolated and permanently ligated using electrocautery under methoxyflurane anesthesia. After a 2-h recovery period with the dam, unanesthetized rats were exposed to hypoxia (humidified $8 \% \mathrm{O}_{2}, 92 \% \mathrm{~N}_{2}, 90 \mathrm{~min}, 36^{\circ} \mathrm{C}$ ambient temperature). Three of 46 pups died during hypoxic exposure $(6.5 \%$ mortality). After hypoxia, litters were culled to 10 pups (23) and assigned to one of two treatment groups: Epo treated $(n=15)$ versus placebo treated $(n=$ 15). $\mathrm{rEpo}\left(2500 \mathrm{U} \cdot \mathrm{kg}^{-1} \cdot \mathrm{d}^{-1}\right.$ s.c.) was prepared in $10 \mathrm{mM}$ PBS vehicle that contained $0.1 \%$ BSA and administered daily for $3 \mathrm{~d}$. Placebo-treated animals were dosed using the same schedule of vehicle injections. Sham animals $(n=$ 10 ) underwent surgery with simple incision but without ligation or hypoxia and received either vehicle $(n=5)$ or Epo $(n=5)$ treatment. Sham groups were collapsed because there was neither injury nor differences in weight, complete blood counts, behaviors, or immunohistochemistry.

Neurodevelopmental behavioral assessment. To identify asymmetrical behaviors that could implicate underlying unilateral neurologic injury, we tested for contralateral sensory neglect and DA agonist-induced behavioral asymmetry. In this model, these phenomena should not be interpreted to suggest that injury is limited to DA neurotransmission or DA receptorcontaining neurons.

On P27, sensorimotor responses to tactile stimulation were scored using an established scale. Briefly, animals were habituated to the testing area, and then a single paintbrush bristle was used to touch the animal lightly across 11 defined areas/hemispheres. This sensory stimulus triggers motor orientation toward the stimulus that can be scored from zero to four as follows: $0=$ none, $1=$ minimal, $2=$ turning halfway, $3=$ complete turning, and $4=$ complete turning and biting the bristle $(24,25)$. A ratio to represent differences between hemispheres then is calculated by summing the scores for each hemisphere and dividing the contralateral by the ipsilateral sum. Ratios less than one indicate impairment of responding to stimulation contralateral to the injury. This phenomenon is termed sensory neglect and is a reliable marker of unilateral brain injury $(24,25)$.

On P28, DA agonist-induced rotation was assessed. Rat pups were placed in individual round chambers and videotaped before and after injection of the
DA receptor agonist apomorphine $(0.2 \mathrm{mg} / \mathrm{kg}$ s.c.). The tape was reviewed, and all rotations ipsilateral (negative) and contralateral (positive) to injury were counted for calculation of net turning in 5-min increments by a blinded observer. The range of number of rotations (ipsilateral to contralateral) is also reported when significance was present.

Safety. Rat pups were weighed on P7, P10, P28, and P30. Blood was obtained at the time they were for evaluation of the complete blood count indices hematocrit, white blood cell count, and platelet count, along with the neutrophil (polymorphonuclear) counts from the white blood cell differential count.

Gross brain injury. Rat pups were killed on P30 with an overdose of pentobarbital (Abbott Laboratories, Chicago, IL) $100 \mathrm{mg} / \mathrm{kg}$ i.p. followed by transcardiac perfusion with heparinized $10 \mathrm{mM}$ PBS followed by $4 \%$ paraformaldehyde. Brains were photographed before paraffin embedding for evaluation of gross brain injury. Gross brain injury was scored using an ordinal scale developed by Vannucci et al. (26) as follows: $0=$ normal ipsilateral hemisphere, $1=$ mild atrophy with $<25 \%$ ipsilateral/contralateral size difference, $2=$ moderate atrophy $\sim 25-50 \%$ difference, $3=$ atrophy with cystic cavitation $<3 \mathrm{~mm}$, and $4=$ atrophy with cystic cavitation $\geq 3 \mathrm{~mm}$.

Immunohistochemistry. DA-containing neurons of the SNpc and VTA were identified by labeling of the catecholamine synthesis enzyme tyrosine hydroxylase $(\mathrm{TH})$. Coronal brain blocks $3 \mathrm{~mm}$ thick were embedded in paraffin, cut to $5 \mu \mathrm{m}$, and mounted on slides. Slides were processed through xylene and declining alcohol concentrations, boiled in $10 \mathrm{mM}$ citrate buffer $(\mathrm{pH} 6)$, and rinsed in 3\% hydrogen peroxide followed by 5\% normal blocking serum. The primary antibody, polyclonal rabbit anti-TH 1:200 (AB-152; Chemicon, Temecula, CA), was applied for $1 \mathrm{~h}$ at $36^{\circ} \mathrm{C}$. A biotinylated secondary antibody (KPL 16-15-06) was applied at 1:200 for $1 \mathrm{~h}$ at room temperature. Avidin-horseradish peroxidase conjugate (Vectastain, Vector Labs, Burlingame, CA) and Fast DAB (D-4168; Sigma Chemical Co., St. Louis, MO) were used to mark immunolabeled cells, and slides were counterstained with hematoxylin. A minimum of three sections per animal were stained. Using a light microscope and $\times 100$ magnification, digital images in each hemisphere of both SNpc and VTA were captured by a blinded observer (27). Using computer software (Soft Imaging System AnalySIS, Ascend Instruments, Portland, OR), images were analyzed by first outlining separately the area of the dorsal tier of the SNpc and VTA (28), then all TH-positive cells within each area were counted. Both areas and cell counts were measured. All measurements from each individual were averaged so that each subject was represented by a single score per variable. Cell densities (count/area) were calculated for both ipsilateral and contralateral SNpc and VTA, and a ratio of ipsilateral/contralateral effect was calculated for subsequent group averages and comparisons. Data are reported as a ratio of ipsilateral/contralateral $\mathrm{TH}$ density for rEpo-, placebo- or sham-treated groups, and mean ipsilateral TH-positive cell counts \pm SEM are also reported.

Statistical analysis. Data were expressed as mean \pm SEM. Differences between placebo-, sham-, and rEpo-treated animals were compared using ANOVA. When significance was present, post hoc tests were performed using Tukey's for between-group comparisons and Dunnett's for within-group comparisons. Differences between proportions were compared using $\chi^{2}$. A $p \leq$ 0.05 was considered significant. Statistics were performed using SPSS software (Chicago, IL).

\section{RESULTS}

Unilateral HI exposure produced varying degrees of gross brain injury both within and between neonatal treatment groups. Variability in gross brain injury is evident in Fig. 1, which shows whole brains photographed from one litter of experimental rat pups that were killed at P30 (Fig. 1A) and the quantified gross injury scores for each treatment group (Fig. 1B). One hundred percent of sham animals but only $40 \%$ of placebo-treated animals were uninjured and given a score of 0 , and this difference indicates that $\mathrm{HI}$ exposure induces significant unilateral gross injury $(p \leq 0.05)$. On this measure, the mean injury score for rEpo-treated animals was $1.1 \pm 0.4$, compared with $1.5 \pm 0.4$ for placebo-treated animals $(p=0.15)$.

Neonatal HI altered the sensorimotor responses to tactile stimulation measured in juvenile rats. Figure 2 shows the sensory neglect ratios determined at P27 in each treatment group. Placebo-treated animals exhibited significantly more sensory neglect than either sham $(p \leq 0.001)$ or rEpo-treated 
A
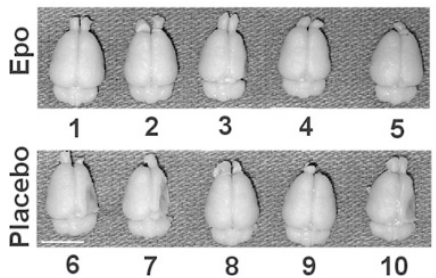

B

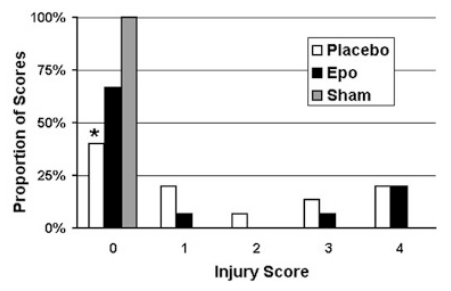

Figure 1. Severity of gross brain injury. Photograph $(A)$ and histogram $(B)$ showing subtle differences in gross brain injury between conditions. Fixed P30 rat brains were scored for increasing injury from 0 to 4 . (A) One litter of Epo-treated (1-5) vs placebo-treated animals (6-10) is shown. Large infarcts are apparent in animals 6, 7, and 10 and a smaller one in animal 3. (B) The frequency distribution for injury scores for all animals is shown. The placebotreated group exhibited significantly fewer uninjured brains (score $=0$ ) when compared with shams $\left({ }^{*} p \leq 0.05\right)$, but the proportion of uninjured scores for Epo-treated rats was not different from any group. Bar $=10 \mathrm{~mm}$.

rats $(p \leq 0.05)$. Although neonatal rEpo treatment reduced the degree of contralateral sensory neglect, it did not prevent it altogether $(p \leq 0.05)$.

Neonatal HI produced asymmetric rotation behavior in juvenile rats that were given apomorphine injection. Figure 3 illustrates the time course of rotation across treatment groups. Placebo-treated animals showed significant ipsilateral rotation 15 min after apomorphine injection compared with sham controls $(p \leq 0.01)$, indicating a functional DA receptor imbalance between right and left hemispheres. In contrast, neither shamnor rEpo-treated animals exhibited asymmetric rotation.

$\mathrm{HI}$-induced loss of dopaminergic neurons in both the SNpc and the VTA was reduced by rEpo treatment. Figure 4 shows photomicrographs of TH staining in the ipsilateral SNpc (Figure $4 A$ ), and a schematic illustrating the sampling areas for SNpc and VTA outlined (Figure $4 B$ ). In Figure $4 C$, neonatal rEpo treatment prevented the HI-induced loss of both SNpc and VTA TH-positive neurons seen after HI treatment compared with placebo-treated animals $(p \leq 0.01)$. Mean ipsilateral TH-positive neuron counts in the SNpc are $62.6 \pm 9.0$, $50.2 \pm 5.7$, and $40.6 \pm 4.7$, respectively, for sham-, rEpo-, and placebo-treated groups. A similar phenomenon existed for the VTA, with mean ipsilateral TH-positive neuron counts of $147.5 \pm 33.7,135.7 \pm 16.9$, and 109.3 \pm 18.5 , respectively, for sham-, rEpo-, and placebo-treated groups. Mean THpositive counts in the SNpc or VTA of the hemisphere contralateral to the injury did not differ between groups (data not shown). A few general observations are worthwhile. In the injured hemisphere of placebo-treated animals, the size of both the SNpc and the VTA and the TH-positive neuron count declined substantially. However, that reduction in area was not sufficient to offset the loss of DA neurons, and thus the ipsilateral/contralateral TH density ratio for placebo-treated animals is still well below one. In comparison, rEpo-treated animals exhibited a small decrease in SNpc and VTA combined with a relative preservation in TH-positive neuron count ipsilateral to the injury; therefore, the ipsilateral/ contralateral density ratio was slightly greater than one. As indicated, sham animals exhibited equivalent TH densities between hemispheres.

Table 1 lists the animal weights and complete blood cell indices collected to assess safety and overall health. Although

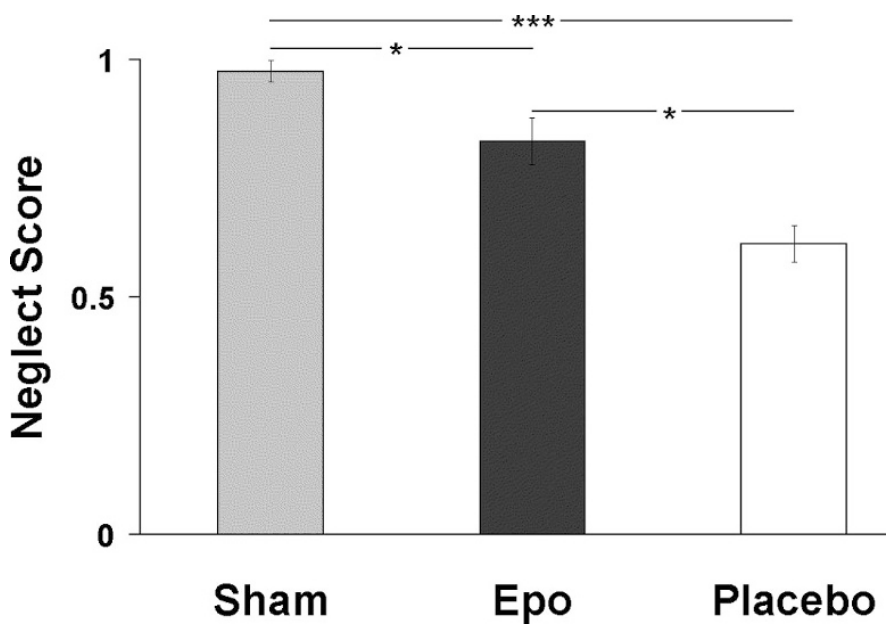

Figure 2. Histogram illustrating differences in sensory neglect scores between conditions. At P27, rats were tested for somatosensory orientation to tactile stimulation and scored for degree of response. ANOVA indicated significant effects of treatment $\left(F_{2,39}=17.5, p<0.001, n=10-15 /\right.$ group $)$. Post hoc testing identified that neonatal unilateral carotid artery occlusion with hypoxia exposure produced sensory neglect compared with shams, and Epo treatment reduced the degree of sensory neglect compared with placebo-treated animals as indicated $(* p \leq 0.05, * * * p \leq 0.001)$.

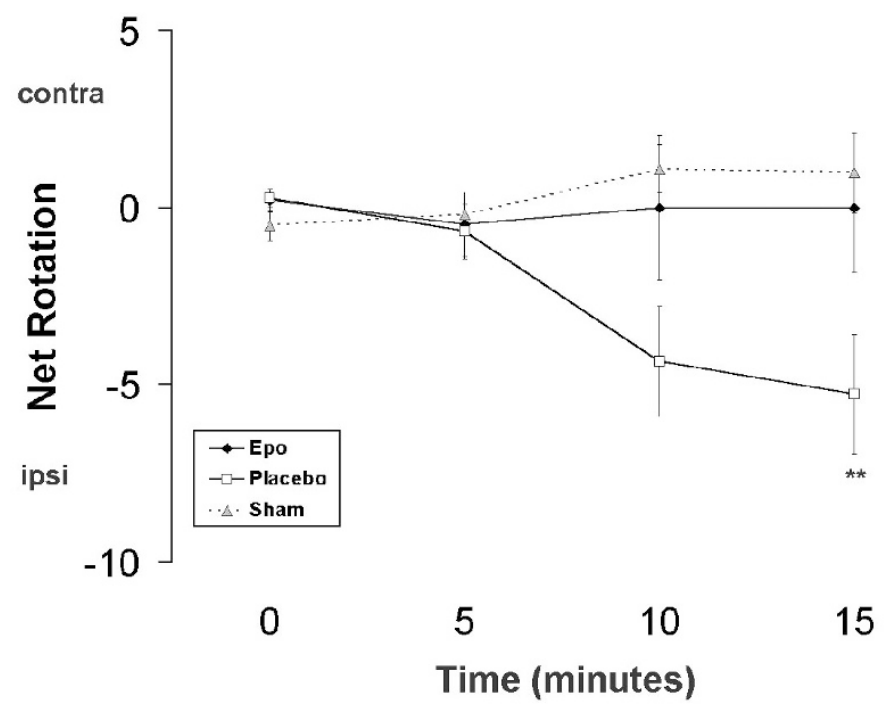

Figure 3. Apomorphine-induced rotation. This graph illustrates the time course of DA agonist-induced rotation. At P28, rats received an injection of apomorphine $(0.2 \mathrm{mg} / \mathrm{kg}$, s.c. $)$, and behavior was filmed for calculation of net rotation. ANOVA identified treatment effects $\left(F_{6,39}=3.3, p \leq 0.01, n=\right.$ 10-15/group). Post hoc testing revealed that placebo-treated rotation differed when compared with Epo- or sham-treated animals $(* * p \leq 0.01)$. Fifteen minutes after injection, placebo-treated animals were rotating ipsilateral to the injury, and Epo prevented manifestation of rotational asymmetry. The maximum rotations during each interval were \pm 3 , $\pm 8,22$, and -18 , respectively.

sham controls weighed more than both groups of HI-exposed animals $(p \leq 0.05)$, there was no weight difference between rEpo- and placebo-treated animals. Last, there were no differences in any parameter of the complete blood cell count among sham-, rEpo-, or placebo-treated animals.

\section{DISCUSSION}

This report extends our understanding of neonatal unilateral hypoxic-ischemic brain injury and identifies neuroprotection 

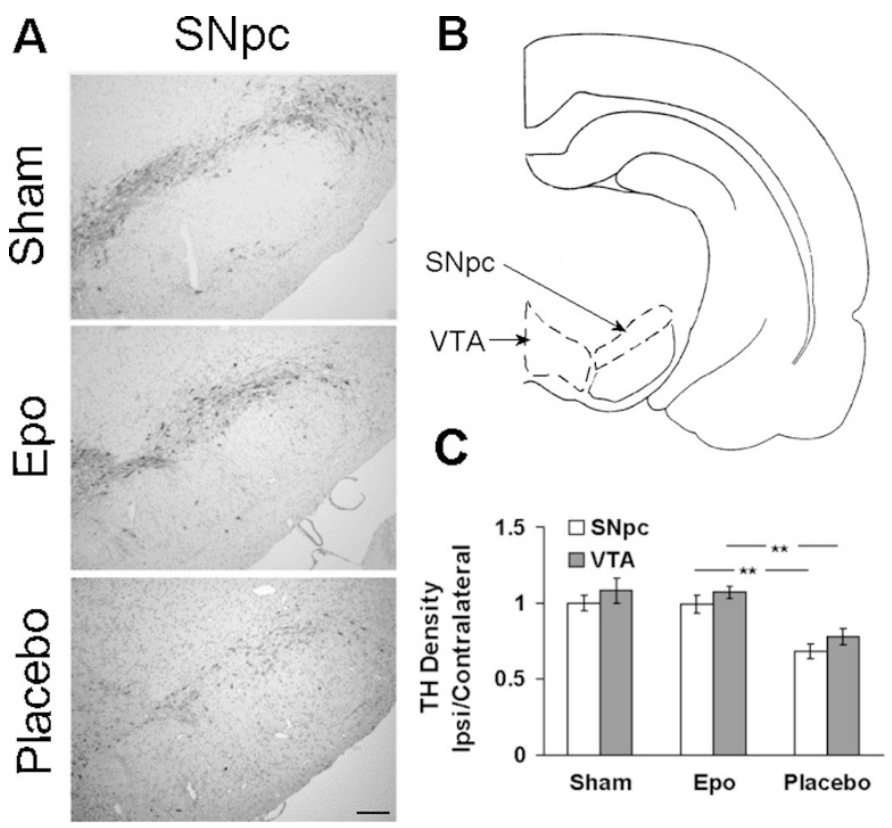

Figure 4. TH immunostaining in dopaminergic mesencephalic nuclei. Quantification of TH immunostaining $3 \mathrm{wk}$ after neonatal $\mathrm{HI}$ exposure revealed differences in the density of DA neurons in the injured hemisphere of both the $\mathrm{SNpc}$ and VTA. (A) Photomicrographs $(\times 100)$ show only the injured SNpc of representatives from each group. $(B)$ Mesencephalic regions quantified. $(C)$ corresponding TH density ratios (ipsilateral/contralateral) between all treatment groups are shown. ANOVA (SNpc, $F_{2,29}=10.1, n=5-14$ /group; VTA, $\mathrm{F}_{2,19}=10.6, n=$ 5-8/group) and subsequent Tukey's post hoc testing found that Epo treatment prevented the substantial unilateral decrease in $\mathrm{TH}$ density seen in placebo-treated animals in both the SNpc and the VTA $(* * p \leq 0.01)$. Bar $=200 \mu \mathrm{m}$.

Table 1. Growth and hematology

\begin{tabular}{lccccc}
\hline Group & $\begin{array}{c}\text { Weight } \\
(\mathrm{g})\end{array}$ & $\begin{array}{c}\mathrm{HCT} \\
(\%)\end{array}$ & $\begin{array}{c}\text { WBC } \\
(1000 / \mu \mathrm{L})\end{array}$ & $\begin{array}{c}\text { PMN } \\
(1000 / \mu \mathrm{L})\end{array}$ & $\begin{array}{c}\text { PLT } \\
(1000 / \mu \mathrm{L})\end{array}$ \\
\hline Sham & 96.6 & 37.3 & 1.41 & 0.25 & 925 \\
Epo & $85.7^{*}$ & 37.3 & 1.82 & 0.34 & 929 \\
Placebo & $87.2^{*}$ & 36.4 & 2.26 & 0.45 & 943 \\
\hline
\end{tabular}

CBC, complete blood count; HCT, hematocrit; WBC, white blood cell count; PMN, neutrophil count; PLT, platelet count.

Although Epo and placebo-treated animals weighed less than shams $\left({ }^{*} p<\right.$ 0.05), no differences were seen in HCT, WBC, PMN or PLT counts between treatment groups.

Twenty-one rats were included in the analysis ( $n=2-11$ per condition) of the $\mathrm{CBC}$.

and corresponding improvement of functional outcomes after early rEpo treatment. By incorporating assessment of behavioral asymmetries associated with DA neurotransmission and sensorimotor function to assess the effectiveness of neonatal rEpo, we have combined two important models toward the common goal of reducing brain injury. Specifically, we demonstrated that high-dose rEpo administered for a brief period after neonatal $\mathrm{HI}$ was able to protect DA neurons, reduce sensory neglect, and prevent rotational asymmetry in the juvenile rat.

In contrast to work published by Kumral et al. (29), we did not see a statistically significant difference in infarct volume between rEpo- and placebo-treated animals. This might be explained by several factors. This neonatal model of unilateral brain injury is known to have variability in the degree of injury, particularly with shorter exposure times to HI (30). This variability within groups might obscure real differences between groups. In the initial presentation of this model of HI-induced brain injury, Rice et al. (2) exposed P7 rat pups to $3.5 \mathrm{~h}$ of hypoxia and subsequently found that $>90 \%$ of animals had evidence of moderate to severe neuronal changes in the ipsilateral cortex and striatum and that $56 \%$ of animals had ipsilateral hemisphere infarctions. Other factors can also affect the degree of brain injury in this model, including carbon dioxide tension (26), energy availability (31), and litter size (23). Given the possibility that a threshold level of injury beyond which no treatment may be possible may exist, we chose a 90-min HI exposure to avoid extensive injury and thus optimize our chance of identifying subtle effects. The dose of rEpo, the dosing schedule, and the timing at which evaluation was done might also account for the differences that we report compared with Kumral et al. (29).

Neonates who are exposed to hypoxic-ischemic brain injury sustain both acute and chronic morbidity that includes sensory, motor, cognitive, and behavioral systems. The full extent of this injury may not be apparent until school age or later $(32,33)$. Premature neonates are particularly vulnerable to HI exposure and may have a variety of deficits when tested at school entry, including decreased IQ scores; slowed processing speed; altered perceptual organization; and difficulties with reading, math, and spelling (34). In addition, several behavioral problems, including decreased attention span and hyperactivity, both hallmarks of attention-deficit/hyperactivity disorder (ADHD), are common in these at-risk populations.

ADHD is mediated in part by DA and involves a complex interaction among the mesocortical, frontostriatal, and nigrostriatal circuits. Disruption of these circuits accounts for the symptoms found in ADHD: mesocortical DA deficit leads to attentional and executive deficits, striatal hyperdopaminergic state leads to hyperactivity, and nigrostriatal DA disruption impairs the learning of complex motor tasks $(35,36)$. Of particular interest is the circuit composed of the VTA, nucleus accumbens/ventral striatum, medial prefrontal cortex, and subiculum of the hippocampus (35). In patients with ADHD, there are alterations in glutamate, DA, and noradrenergic transmission within this circuit. In addition, the $\mathrm{SNpc}$ has been shown to interact with the VTA and both the ventral and dorsal striatum and thereby has a role in integrating the various basal ganglia circuits (37). We have shown that rEpo administered for $3 \mathrm{~d}$ after neonatal $\mathrm{HI}$ injury was able to preserve the number of mesencephalic DA neurons that persisted into the juvenile period in rats. This preliminary work offers promise that neonatal treatment with rEpo may lessen behavioral disability in HI-exposed neonates.

rEpo was shown previously to have multiple beneficial effects in the CNS, including decreasing glutamate toxicity, inhibiting apoptosis, decreasing oxidative injury, decreasing inflammation, and decreasing blood-brain barrier permeability. Neonatal rEpo after $\mathrm{HI}$ brain injury also was shown recently to preserve adult hippocampal CA1 neuron counts and provide lasting protection against spatial memory deficits (38). rEpo has also been shown to stimulate DA release from the striatum (39), and exposure to HI has been shown to affect both DA receptor expression and DA reuptake transporter expression $(3,40)$. EpoR was identified recently on adult DA neurons (41). The mechanism of rEpo's protection of DA neurons in this study is currently being investi- 
gated in our laboratory. We speculate that it may include a combination of the known actions of Epo within the CNS or may involve other DA-specific mechanisms of Epo. It has been shown that neural progenitors that were exposed to hypoxic conditions differentiated along a dopaminergic pathway (19). In addition, this tendency of neural progenitors to differentiate into dopaminergic neurons was replicated with rEpo treatment of the neural progenitors and blocked by the administration of anti-Epo antibody.

In this study, we not only demonstrated important neuroprotective effects of rEpo, but we also showed that short-term treatment with high-dose rEpo in the neonatal period seemed to be safe. We did not see differences in hematocrit, platelet counts, white blood cell counts, or neutrophil counts between treatment groups. There were differences in weight between sham-treated and rEpo- or placebo-treated animals 3 wk after brain injury but none between rEpo- or placebo-treated animals, suggesting the HI exposure was the likely explanation for the weight differences. We chose a dose of rEpo (2500 units · $\mathrm{kg}^{-1} \cdot \mathrm{d}^{-1}$ s.c.) that results in therapeutic cerebrospinal fluid levels when given to immature animals with intact blood-brain barriers (8), and we administered rEpo during the peak of inflammation in this injury model (42). Recently, rEpo was administered at doses up to 30,000 units $\cdot \mathrm{kg}^{-1} \cdot \mathrm{d}^{-1}$ in animal models (43), and both the timing and the dose of Epo used for neuroprotection continue to be actively investigated.

In summary, we have demonstrated a protective effect of high-dose rEpo on the nigrostrial DA system when administered after neonatal HI. These findings add to the growing body of literature supporting the use of rEpo as a neuroprotective agent and demonstrate beneficial effects of rEpo on neurodevelopment when given after neonatal HI brain injury.

\section{REFERENCES}

1. Vannucci RC, Connor JR, Mauger DT, Palmer C, Smith MB, Towfighi J, Vannucci SJ 1999 Rat model of perinatal hypoxic-ischemic brain damage. J Neurosci Res 55: $158-163$

2. Rice JE 3rd, Vannucci RC, Brierley JB 1981 The influence of immaturity on hypoxic-ischemic brain damage in the rat. Ann Neurol 9:131-141

3. Meng SZ, Ohyu J, Itoh M, Takashima S 2000 Dopamine transporter and nitric oxide synthase in hypoxic-ischemic brain. Pediatr Neurol 22:115-121

4. Bauer R, Brust P, Walter B, Vorwieger G, Bergmann R, Elhalag E, Fritz A, Steinbach J, Fuchtner F, Hinz R, Zwiener U, Johannsen B 2002 Effect of hypoxia/hypercapnia on metabolism of 6-[(18)F]fluoro-L-DOPA in newborn piglets. Brain Res 934:23-33

5. Juul S 2002 Erythropoietin in the central nervous system, and its use to prevent hypoxic-ischemic brain damage. Acta Paediatr Suppl 91:36-42

6. Buemi M, Cavallaro E, Floccari F, Sturiale A, Aloisi C, Trimarchi M, Corica F, Frisina N 2003 The pleiotropic effects of erythropoietin in the central nervous system. J Neuropathol Exp Neurol 62:228-236

7. Grasso G, Sfacteria A, Cerami A, Brines M 2004 Erythropoietin as a tissue-protective cytokine in brain injury: what do we know and where do we go? Neuroscientist 10:93-98

8. Juul SE, McPherson RJ, Farrell FX, Jolliffe L, Ness DJ, Gleason CA 2004 Erythropoietin concentrations in cerebrospinal fluid of nonhuman primates and fetal sheep following high-dose recombinant erythropoietin. Biol Neonate 85:138-144

9. Morishita E, Masuda S, Nagao M, Yasuda Y, Sasaki R 1997 Erythropoietin receptor is expressed in rat hippocampal and cerebral cortical neurons, and erythropoietin prevents in vitro glutamate-induced neuronal death. Neuroscience 76:105-116

10. Sinor AD, Greenberg DA 2000 Erythropoietin protects cultured cortical neurons, but not astroglia, from hypoxia and AMPA toxicity. Neurosci Lett 290:213-215

11. Chong ZZ, Kang JQ, Maiese K 2003 Erythropoietin fosters both intrinsic and extrinsic neuronal protection through modulation of microglia, Akt1, Bad, and caspase-mediated pathways. Br J Pharmacol 138:1107-1118

12. Alafaci C, Salpietro F, Grasso G, Sfacteria A, Passalacqua M, Morabito A, Tripodo E, Calapai G, Buemi M, Tomasello F 2000 Effect of recombinant human erythropoietin on cerebral ischemia following experimental subarachnoid hemorrhage. Eur J Pharmacol 406:219-225

13. Digicaylioglu M, Lipton SA 2001 Erythropoietin-mediated neuroprotection involves cross-talk between Jak2 and NF- $\kappa$ B signalling cascades. Nature 412:641-647

14. Kumral A, Baskin H, Gokmen N, Yilmaz O, Genc K, Genc S, Tatli MM, Duman N, Ozer E, Ozkan H 2004 Selective inhibition of nitric oxide in hypoxic-ischemic brain model in newborn rats: is it an explanation for the protective role of erythropoietin? Biol Neonate 85:51-54

15. Martinez-Estrada OM, Rodriguez-Millan E, Gonzalez-De Vicente E, Reina M, Vilaro S, Fabre M 2003 Erythropoietin protects the in vitro blood-brain barrier against VEGF-induced permeability. Eur J Neurosci 18:2538-2544

16. Sugawa M, Sakurai Y, Ishikawa-Ieda Y, Suzuki H, Asou H 2002 Effects of erythropoietin on glial cell development; oligodendrocyte maturation and astrocyte proliferation. Neurosci Res 44:391-403

17. Shingo T, Sorokan ST, Shimazaki T, Weiss S 2001 Erythropoietin regulates the in vitro and in vivo production of neuronal progenitors by mammalian forebrain neural stem cells. J Neurosci 21:9733-9743

18. Koshimura K, Murakami Y, Sohmiya M, Tanaka J, Kato Y 1999 Effects of erythropoietin on neuronal activity. J Neurochem 72:2565-2572

19. Studer L, Csete M, Lee SH, Kabbani N, Walikonis J, Wold B, McKay R 2000 Enhanced proliferation, survival, and dopaminergic differentiation of CNS precursors in lowered oxygen. J Neurosci 20:7377-7383

20. Lee JY, Koh HC, Chang MY, Park CH, Lee YS, Lee SH 2003 Erythropoietin and bone morphogenetic protein 7 mediate ascorbate-induced dopaminergic differentiation from embryonic mesencephalic precursors. Neuroreport 14:1401-1404

21. Brines ML, Ghezzi P, Keenan S, Agnello D, de Lanerolle NC, Cerami C, Itri LM, Cerami A 2000 Erythropoietin crosses the blood-brain barrier to protect against experimental brain injury. Proc Natl Acad Sci USA 97:10526-10531

22. Jackson-Lewis V, Vila M, Djaldetti R, Guegan C, Liberatore G, Liu J, O’Malley KL, Burke RE, Przedborski S 2000 Developmental cell death in dopaminergic neurons of the substantia nigra of mice. J Comp Neurol 424:476-488

23. Oakden E, Chiswick M, Rothwell N, Loddick S 2002 The influence of litter size on brain damage caused by hypoxic-ischemic injury in the neonatal rat. Pediatr Res 52:692-696

24. Marshall JF 1979 Somatosensory inattention after dopamine-depleting intracerebral 6-OHDA injections: spontaneous recovery and pharmacological control. Brain Res 177:311-324

25. Marshall JF, Berrios N, Sawyer S 1980 Neostriatal dopamine and sensory inattention. J Comp Physiol Psychol 94:833-846

26. Vannucci RC, Towfighi J, Heitjan DF, Brucklacher RM 1995 Carbon dioxide protects the perinatal brain from hypoxic-ischemic damage: an experimental study in the immature rat. Pediatrics 95:868-874

27. Soriano MA, Justicia C, Ferrer I, Rodriguez-Farre E, Planas AM 1997 Striatal infarction in the rat causes a transient reduction of tyrosine hydroxylase immunoreactivity in the ipsilateral substantia nigra. Neurobiol Dis 4:376-385

28. Paxinos G, Watson C 1986 The Rat Brain in Stereotaxic Coordinates, 2nd Ed. New York, Academic Press

29. Kumral A, Ozer E, Yilmaz O, Akhisaroglu M, Gokmen N, Duman N, Ulukus C, Genc S, Ozkan H 2003 Neuroprotective effect of erythropoietin on hypoxic-ischemic brain injury in neonatal rats. Biol Neonate 83:224-228

30. Towfighi J, Mauger D, Vannucci RC, Vannucci SJ 1997 Influence of age on the cerebral lesions in an immature rat model of cerebral hypoxia-ischemia: a light microscopic study. Brain Res Dev Brain Res 100:149-160

31. Yager JY, Brucklacher RM, Vannucci RC 1992 Cerebral energy metabolism during hypoxia-ischemia and early recovery in immature rats. Am J Physiol 262:672-677

32. Volpe JJ 2001 Perinatal brain injury: from pathogenesis to neuroprotection. Ment Retard Dev Disabil Res Rev 7:56-64

33. du Plessis AJ, Volpe JJ 2002 Perinatal brain injury in the preterm and term newborn. Curr Opin Neurol 15:151-157

34. Anderson P, Doyle LW; Victorian Infant Collaborative Study Group 2003 Neurobehavioral outcomes of school-age children born extremely low birth weight or very preterm in the 1990s. JAMA 289:3264-3272

35. Russell VA 2003 Dopamine hypofunction possibly results from a defect in glutamatestimulated release of dopamine in the nucleus accumbens shell of a rat model for attention deficit hyperactivity disorder - the spontaneously hypertensive rat. Neurosci Biobehav Rev 27:671-682

36. Sullivan RM, Brake WG 2003 What the rodent prefrontal cortex can teach us about attention-deficit/hyperactivity disorder: the critical role of early developmental events on prefrontal function. Behav Brain Res 146:43-55

37. Haber SN, Fudge JL, McFarland NR 2000 Striatonigrostriatal pathways in primates form an ascending spiral from the shell to the dorsolateral striatum. J Neurosci 20:2369-2382

38. Kumral A, Uysal N, Tugyan K, Sonmez A, Yilmaz O, Gokmen N, Kiray M, Genc S, Duman N, Koroglu TF, Ozkan H, Genc K 2004 Erythropoietin improves long-term spatial memory deficits and brain injury following neonatal hypoxia-ischemia in rats. Behav Brain Res 153:77-86

39. Yamamoto M, Koshimura K, Kawaguchi M, Sohmiya M, Murakami Y, Kato Y 2000 Stimulating effect of erythropoietin on the release of dopamine and acetylcholine from the rat brain slice. Neurosci Lett 292:131-133

40. Labaune JM, Boutroy MJ, Bairam A 2003 Age-related modulation of dopamine d1 receptor mRNA level by hypoxia in rabbit adrenal gland. Biol Neonate 83:217-223

41. Csete M, Rodriguez L, Wilcox M, Chadalavada S 2004 Erythropoietin receptor is expressed on adult rat dopaminergic neurons and erythropoietin is neurotrophic in cultured dopaminergic neuroblasts. Neurosci Lett 359:124-126

42. Cowell RM, Xu H, Galasso JM, Silverstein FS 2002 Hypoxic-ischemic injury induces macrophage inflammatory protein- $1 \alpha$ expression in immature rat brain. Stroke 33:795-801

43. Dzietko M, Felderhoff-Mueser U, Sifringer M, Krutz B, Bittigau P, Thor F, Heumann R, Buhrer C, Ikonomidou C, Hansen HH 2004 Erythropoietin protects the developing brain against $\mathrm{N}$-methyl-D-aspartate receptor antagonist neurotoxicity. Neurobiol Dis 15:177-187 